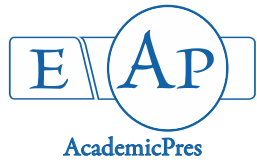

\title{
Effect of Light Quality, Sucrose and Trehalose on In Vitro Organogenesis of Cymbidium devonianum (Lindl.)
}

\author{
Syed M. HAQUE ${ }^{1}$, Syeda J. NAHAR ${ }^{2 *}$, Kazuhiko SHIMASAKI ${ }^{2}$ \\ ${ }^{1}$ Ehime University, The United Graduate School of Agricultural Sciences, 3-5-7 Tarumi, Matsuyama, 790-8556 Ehime, \\ Japan; guddu7771@gmail.com \\ ${ }^{2}$ Kochi University, Faculty of Agriculture, B200 Monobe, Nankoku, 783-8502 Kochi, \\ Japan; naharmunna@yahoo.com ( ${ }^{*}$ correspondingauthor); shim@kochi-u.ac.jp
}

\begin{abstract}
The present study investigated the effect of sucrose, trehalose and combination of sucrose and trehalose with modified MS medium on in vitro regulation of protocorm-like bodies (PLBs) of Cymbidium devonianum under different quality of lights (white fluorescent tube, green, red and blue LED). As a result of this study, new PLB and shoots were successfully regenerated on modified MS medium under different quality of lights. The highest PLB formation rate (100\%) and the highest shoot formation rate (85\%) were observed amongst explants cultured on medium supplemented with $10 \mathrm{~g} / \mathrm{l}$ sucrose $+10 \mathrm{~g} / \mathrm{l}$ trehalose under green LED. The maximum fresh weight of PLBs, the highest average number of PLBs and shoots were recorded on medium containing $10 \mathrm{~g} / \mathrm{l}$ sucrose $+10 \mathrm{~g} / \mathrm{l}$ trehalose under green LED. For plant tissue culture, sucrose is considered an indisputably important carbon and energy source and biosynthesis of trehalose is similar to that of sucrose. The hereby study concluded that the contribution of LED lights, sucrose and trehalose (combined) can induce PLB and shoot formation of Cymbidium devonianum tissue culture without the use of any other plant growth regulator, whereas the green light showed the best formation rate compare with the other studied qualities of lights.
\end{abstract}

Keywords: energy efficient light source, LED, organogenesis, PLBs, tissue culture

\section{Introduction}

Orchids belong to the family Orchidaceae and are one of the largest and most evolved flowering plants. Cymbidium devonianum (Lindl.) is an epiphytic orchid, relatively rare and considered a threatened orchid species (Das et al., 2011). This species is used in the breeding programs of many miniature hybrids. The growers greatly recognize this promiscuity because it allows them to endlessly produce new combinations of colour and pattern.

In vitro micropropagation techniques are useful for the propagation and maintenance of large numbers of threatened plant species (Dhar et al., 2000). Moreover, Cymbidium is rapidly turning into a model orchid, if not a model plant, at least for in vitro studies, due to the fine scale nature of experiments conducted on this genus (Teixeira da Silva, 2014). Hence, an effective strategy becomes essential to salvage and multiply this species.

Plants are able to modify their growth, development and physiology according to a variable environment. Light is one of the most important abiotic factors that influences the successful establishment and subsequent development of a plant culture. Recently, light-emitting diodes (LED) have been developed for accelerating plantlet growth. LEDs can play a variety of roles in horticultural lighting. They are particularly well suited for research applications (e.g., in growth chambers) as a result of their unique capabilities and lend themselves well to shelf lighting for tissue culture applications as a result of their low profile and low radiant heat output (Bula et al., 1991; Barta et al., 1992; Morrow, 2008). The effects of LEDs on plantlets cultured in vitro were reported in many plants such as potato (Miyashita et al., 1995), Cymbidium (Tanaka et al., 1998; Nahar et al., 2012, 2013; Kaewjampa and Shimasaki, 2012), Dendrobium (Sultana et al., 2014), Rehmannia glutinose (Hahn et al., 2000) and strawberry (Nhut et al., 2003). The reaction to different lighting conditions differs between species and their various growth stages.

Another important component in plant tissue culture media is the carbon source, because they supply energy to the plants, especially when they are not ready to photosynthesize their own food during the early stage of tissue culture (AlKhateeb, 2008). The growth of the plants is also influenced by the presence of carbon source. Carbon source can be in the form of simple or complex sugars (Akter et al., 2007). 
90

Normally, sucrose is used as the carbon source in plant tissue culture. In the current study, sucrose and trehalose were used as the carbon source within different medium. It is advisable to use organic additives in orchid culture medium as this has been reported to be an easy way to improvise the current plant tissue culture media towards commercial production (Ichihashi and Islam, 1999). Trehalose (a,a-trehalose or a-D-glucopyranosyl aD-glucopyranoside), considered to be more effective than others such as proline, is a nonreducing disaccharide of glucose that occurs in several organisms such as bacteria, fungi, nematodes and crustaceans (Elbein, 1974). It stabilizes proteins and membranes under stress conditions, especially under desiccation and heat stress, preventing denaturation of proteins and the fusion of membranes (Wingler, 2002). Trehalose also plays a major role in plant carbohydrate metabolism (Eastmond et al., 2003). Very few studies have been done on using trehalose as a substitute for carbon source for the proliferation of PLBs of Cymbidium orchid.

The aim of the hereby study was to investigate the effect of the addition of sucrose and trehalose as carbon source on the growth and proliferation of protocorm-like bodies (PLBs) of Cymbidium devonianum under different sources of lights.

\section{Materials and Methods}

\section{Plant material and explants source}

Protocorm-like bodies (PLB) of Cymbidium devonianum were proliferated in the modified Murashige and Skoog (Shimasaki and Uemoto, 1990) medium by transfering new medium every two months. After excision of PLB (ca. $5 \mathrm{~mm}$ in diameter) into pieces, they were used as explants.

\section{Media and culture conditions}

Modified MS medium were used for culture; MS medium with $412.5 \mathrm{mg} \mathrm{L}^{-1}$ ammonium nitrate, $950 \mathrm{mg} \mathrm{L}^{-1}$ potassium nitrate, $20 \mathrm{gL}^{-1}$ sucrose $/ 20 \mathrm{gL}^{-1}$ trehalose $/ 10 \mathrm{~g} \mathrm{~L}^{-1}$ sucrose $+10 \mathrm{~g}$ $\mathrm{L}^{-1}$ trehalose, and $2 \mathrm{gL}^{-1}$ Phytagel (Sigma) were used. The $\mathrm{pH}$ of the medium was adjusted to $5.5-5.8$ using $0.1 \mathrm{mM}$ 2-(Nmorpholino) ethanesulfonic acid sodium salt (MES-Na) before autoclaved at $121^{\circ} \mathrm{C}$ for $15 \mathrm{~min}$. Jars of $250 \mathrm{ml}$ (UM culture bottle) with plastic caps were used, each bottle receiving $30 \mathrm{ml}$ of medium. Five explants were cultured in each culture vessel and four culture vessels were used for each treatment.

\section{Light treatments}

The cultures of in vitro plantlets were illuminated using different light conditions of photon flux density (PFD) of 50 $\mu$ mole $\mathrm{m}^{-2} \bullet \mathrm{s}^{-1}$. Four sources of light were used: white fluorescent tube (National FL20SS), blue LED (Jefcom, P18W-E1701-B, peak wavelength: $450 \mathrm{~nm}$ ), red LED (Jefcom, P18W-E1701-R, peak wavelength: $640 \mathrm{~nm}$ ) and green LED (Jefcom, P18WE1701-G, peak wavelength: $510 \mathrm{~nm}$ ). The plantlets were exposed to a $16 \mathrm{~h}$ photoperiod for $5 / 6$ weeks.

\section{Statistical analyses}

Experimental data were collected after $5 / 6$ weeks of culture by counting the number of PLB and shoot; percentage of PLB and shoot and the fresh weight of PLB were measured. The data were analysed to a one-way analysis variance (ANOVA) and differences between means were tested using Tukey's honestly significant different test $(\mathrm{P} \leq 0.05)$.

\section{Results and Discussion}

In vitro culture plays important roles in the propagation of plants in large quantities with desired characters, and has also been used as a tool for the conservation and rapid propagation of rare and endangered plants of economic importance (Gupta and Jatothu, 2013).

The illumination system for in vitro culture should provide light in the spectral region that is involved in photosynthesis and in the photomorphogenic responses (Bula et al., 1991; Seabrook, 2005). In the recent past, several studies have been conducted on the effect of LED lights on orchid tissue culture using sucrose as a carbon source within in vitro medium (Sultana et al., 2014; Nahar et al., 2015; Haque et al., 2016). Nahar et al. (2014) reported that trehalose and hyaluronic acid added with culture medium rapidly increased the formation of PLB, shoot and root of Cymbidium spp. under white fluorescent tube.

In vitro culture of PLBs in modified MS medium supplemented with sucrose under different sources of lights

The effects of sucrose with modified MS medium on organogenesis on PLB culture of $C$. devonianum are shown in Table 1. Significant differences on PLB culture of $C$. devonianum under green LED compare with other three sources of lights were observed. A great (100\%) PLB formation rate was observed when PLBs were cultured on modified MS medium under green LED and 90\% PLB formation rate was observed with the other three different (white fluorescent tube, red LED and blue LED) sources of lights. The highest number of PLBs (15.1 PLBs/explant) and maximum fresh weight $122.3 \mathrm{mg}$ were observed under green LED. The maximum shoot formation $45 \%$ and the highest number of shoots $(0.5$ shoots/ explant) were observed under white fluorescent tube after 6 weeks of culture.

These results were supported with previous data on $C$. insigne (Nahar et al., 2012), whereas the average number of PLBs, maximum formation of PLB and fresh weight of PLBs were higher under green LED, and shoot formation increased under white fluorescent tube. Sultana et al. (2014) reported that blue and red LED plays an important role on organogenesis of Dendrobium kingianum, while sucrose was used as a carbon sources with medium.

Table 1. In vitro culture of $C$. devonianum PLBs in modified MS medium supplemented with sucrose $\left(20 \mathrm{~g} \mathrm{~L}^{-1}\right.$ with modified MS medium) under different sources of lights

\begin{tabular}{|c|c|c|c|c|c|}
\hline \multirow{2}{*}{$\begin{array}{l}\text { Light } \\
\text { sources }\end{array}$} & \multicolumn{2}{|c|}{ PLBs } & \multicolumn{2}{|c|}{ Shoot } & \multirow{2}{*}{$\begin{array}{c}\text { Fresh } \\
\text { weight }\end{array}$} \\
\hline & No./Explants & Rate (\%) & No./Explants & Rate (\%) & \\
\hline White F. Tube & $5.6 \pm 0.7 c$ & 90 & $0.5 \pm 0.2 \mathrm{a}$ & 45 & $27.3 \pm 2.9 c$ \\
\hline Green LED & $15.1 \pm 2.1 \mathrm{a}$ & 100 & $0.2 \pm 0.2 \mathrm{a}$ & 20 & $122.3 \pm 11.2 \mathrm{a}$ \\
\hline Red LED & $13.4 \pm 2.0 \mathrm{ab}$ & 90 & $0.4 \pm 0.2 \mathrm{a}$ & 30 & $110.1 \pm 18.1 \mathrm{ab}$ \\
\hline Blue LED & $7.9 \pm 1.7 \mathrm{bc}$ & 90 & $0.3 \pm 0.2 \mathrm{a}$ & 15 & $53.7 \pm 8.1 c$ \\
\hline
\end{tabular}


In vitro culture of PLBs in modified $M S$ medium supplemented with trehalose under different sources of lights

The effects of trehalose with modified MS medium on organogenesis on PLB culture of $C$. devonianum are shown in Table 2. A great (100\%) PLB formation rate was observed when PLBs were cultured on modified MS medium under green LED and red LED and 90\% PLBs formation rate was observed under white fluorescent tube and blue LED. Significant differences were observed under green and red LED. The highest number of PLBs (18.1 PLBs/explant) was observed when PLBs were cultured under red LED and medium contain $20 \mathrm{~g} / \mathrm{l}$ trehalose. The maximum fresh weight $150.8 \mathrm{mg}$ and the maximum shoot formation rate $30 \%$ were observed under green LED after 6 weeks of culture. The maximum numbers of shoot ( 0.3 shoots/ explant) were recorded when PLBs were cultured under green and red LED.

In plant tissue culture, sucrose is considered for being an indisputably important carbon and energy source because sucrose is the most common carbohydrate in phloem sap and is involved in controlling developmental processes (Gibson, 2000). In spite of the fact that the biosynthesis of trehalose is similar to that of sucrose, its evolutionary origin is probably more ancient because it is present in all kingdoms (Elbein, 1974). With trehalose added with modified MS medium, Cymbidium dayanum 53\% PLB formation, 47\% shoot formation and $40 \%$ root formation were observed within 3 weeks of culture under white fluorescent tube (Nahar et al., 2014). This study results revealed that trehalose with medium induced 100\% PLB formation under green and red LED within 6 weeks of culture.

In vitro culture of PLBs in modified $M S$ medium supplemented with sucrose + trehalose under different sources of lights

The combination effects of sucrose and trehalose with modified MS medium on organogenesis on PLBs culture of C. devonianum were shown in Table 3. A large (100\%) PLB formation rate was observed when PLBs were cultured on modified MS medium under white fluorescent tube and green LED and $85 \%$ shoot formation rate and 65\% root formation rate was observed under green LED within 5 weeks of culture. PLBs of $C$. devenianum were significantly increased under green LED. The highest number of PLBs (7.6 PLBs/explant), highest number of shoots (1.1 shoots/explant) and highest number of roots (0.7 roots/explant) were observed under green LED. The maximum fresh weight $(113.5 \mathrm{mg})$ was observed under green LED after 5 weeks of culture.

Sucrose syntheses activity has been shown to play a major role in energy metabolism, controlling the mobilization of sucrose into various pathways important for the metabolic, structural and storage functions of the plant cell (Hesse and Willmitzer, 1996; Tang et al., 1999). Trehalose, a nonreducing disaccharide, is found in some organisms including a number of plants in which it serves as an osmoprotectant. It stabilizes proteins and biological membranes under a variety of stress conditions including hydrostatic pressure and osmotic stress (Benaroudi et al., 2001). Other proposed roles of trehalose include regulation of carbohydrate utilization, plant growth and development (Schluepmann et al., 2004).

In Phalaenopsis, blue LEDs proved to be effective for PLB formation from cultured PLB segments (Tanaka et al., 2001). Huan and Tanaka (2004) reported that red LEDs were the most effective light source for inducing callus tissue from PLB. In particular, red and blue lights, either alone or in combination, have a significant influence on plant growth. Folta and Maruhnich (2007) suggested that green light has discrete effects on plant biology, and the mechanisms that sense this light quality are now being elucidated. Generally, the effects of green light are in opposition with those directed by red and blue wavebands. Green light is efficiently transmitted through the plant body, playing more of a role in photosynthesis than red or blue in some contexts (Sun et al., 1998), suggesting green light may prove useful as a signal to tissues not directly exposed to the light environment. Potential green light effects may also vary with developmental context. According to the present study, when sucrose and trehalose were both added with medium, maximum PLB, shoot and root formation occurred within 5 weeks of culture

Table 2. In vitro culture of $C$. devonianum PLBs in modified MS medium supplemented with trehalose $\left(20 \mathrm{~g} \mathrm{~L}^{-1}\right.$ with modified MS medium) under different sources of lights

\begin{tabular}{|c|c|c|c|c|c|}
\hline \multirow{2}{*}{$\begin{array}{l}\text { Light } \\
\text { sources }\end{array}$} & \multicolumn{2}{|c|}{ PLBs } & \multicolumn{2}{|c|}{ Shoot } & \multirow{2}{*}{$\begin{array}{c}\text { Fresh } \\
\text { weight }\end{array}$} \\
\hline & No./Explants & Rate (\%) & No./Explants & Rate (\%) & \\
\hline White F. Tube & $4.9 \pm 0.9 c$ & 90 & $0.2 \pm 0.2 \mathrm{a}$ & 10 & $24.5 \pm 4.6 c$ \\
\hline Green LED & $11.7 \pm 1.8 \mathrm{a}$ & 100 & $0.3 \pm 0.2 \mathrm{a}$ & 30 & $150.8 \pm 47.3 a$ \\
\hline Red LED & $18.1 \pm 1.4 b$ & 100 & $0.3 \pm 0.2 \mathrm{a}$ & 25 & $124.1 \pm 11.6 \mathrm{ab}$ \\
\hline Blue LED & $4.1 \pm 0.4 c$ & 90 & $0.2 \pm 0.2 \mathrm{a}$ & 15 & $29.9 \pm 3.2 \mathrm{c}$ \\
\hline
\end{tabular}

Value represents means \pm SE $(\mathrm{n}=20)$ followed by the different letters which show significant differences by Tukey HSD test $(\mathrm{P} \leq 0.05)$; White F. Tube $=$ White fluorescent tube

Table 3. In vitro culture of $C$. devonianum PLBs in modified MS medium supplemented with sucrose and trehalose $\left(10 \mathrm{~g} \mathrm{~L}^{-1}+10 \mathrm{~g} \mathrm{~L}^{-1}\right.$ with modified MS medium) under different sources of lights

\begin{tabular}{|c|c|c|c|c|c|c|c|}
\hline \multirow{2}{*}{$\begin{array}{c}\text { Light } \\
\text { sources }\end{array}$} & \multicolumn{2}{|c|}{ PLBs } & \multicolumn{2}{|c|}{ Shoot } & \multicolumn{2}{|c|}{ Root } & \multirow[b]{2}{*}{$\begin{array}{c}\text { Fresh } \\
\text { weight }\end{array}$} \\
\hline & $\begin{array}{c}\text { No./ } \\
\text { Explants }\end{array}$ & $\begin{array}{c}\text { Rate } \\
(\%)\end{array}$ & $\begin{array}{c}\text { No./ } \\
\text { Explants }\end{array}$ & Weight & $\begin{array}{c}\text { No./ } \\
\text { Explants }\end{array}$ & $\begin{array}{l}\text { Rate } \\
(\%)\end{array}$ & \\
\hline White F. Tube & $7.4 \pm 0.8 b$ & 100 & $0.7 \pm 0.2 b$ & 60 & $0.4 \pm 0.2$ & 40 & $44.6 c$ \\
\hline Green LED & $7.6 \pm 0.9 \mathrm{a}$ & 100 & $1.1 \pm 0.1 \mathrm{a}$ & 85 & $0.7 \pm 0.2$ & 65 & $113.5 \mathrm{a}$ \\
\hline Red LED & $6.8 \pm 1.3 \mathrm{bc}$ & 85 & $0.7 \pm 0.2 b c$ & 45 & $0.4 \pm 0.2$ & 40 & $85.7 \mathrm{abc}$ \\
\hline Blue LED & $4.9 \pm 0.6 b c$ & 90 & $0.6 \pm 0.1 b c$ & 60 & $0.2 \pm 0.2$ & 15 & $43.7 \mathrm{bc}$ \\
\hline
\end{tabular}

fluorescent tube 
92

under green LED, while individual addition of sucrose and trehalose had no root formation within 6 weeks of culture. Formation of multiple shoots and roots from protocorm-like bodies (PLBs) are very common co-sequential phenomenon in vitro. The subsequent growth and effective shoot and root development on suitable medium play a vital role in orchid production.

\section{Conclusions}

From all the four sources of lights, green LED promoted the maximum proliferation of PLB and shoot of Cymbidium devonianum in vitro and the contribution of LED lights, sucrose and trehalose can induced PLB and shoot formation of Cymbidium devonianum tissue culture without the use of any plant growth regulator. It was highlighted that combination of sucrose and trehalose induced PLB, shoot and root formation within a short duration of culture period, comparatively with individual addition of sucrose and trehalose with the medium.

\section{References}

Akter S, Nasiruddin KM, Khaldun ABM (2007). Organogenesis of Dendrobium orchid using traditional media and organic extracts. Journal of Agriculture and Rural Development 5:30-35.

Al-Khateeb AA (2008). Regulation of in vitro bud formation of date palm (Phoenix dactylifera L.) cv. Khanezi by different carbon sources. Bioresource Technology 99(14):6550-6555.

Barta DJ, Tibbitts TW, Bula RJ, Merrow TW (1992). Evaluation of light-emitting diodes characteristics for a space-based plant irradiation source. Advance in Space Research 12:141-149.

Benaroudj N, Lee DH, Goldberg AL (2001). Trehalose accumulation during cellular stress protects cells and cellular proteins from damage by oxygen radicals. Journal of Biological Chemistry 276:2426124267.

Bula RJ, Morrow TW, Tibbitts TW, Barta DJ, Ignatius RW, Martin TS (1991). Light-emitting diodes as a radiation source for plants. HortScience 120:808-881.

Das MC, Kumaria S, Tandon P (2011). Storage and high conversion frequency of encapsulated protocorm-like bodies of Cymbidium devonianum (orchid). The Journal of Horticultural Science and Biotechnology 86(6):611-615.

Dhar U, Upreti J, Bhatt ID (2000). Micropropagation of Pittosporum napaulensis (DC) Rehder and Wilson-a rare, endemic Himalayan medeicinal tree. Plant Cell Tissue and Organ Culture 63:231-235.

Eastmond PJ, Li Y, Graham IA (2003). Is trehalose-6-phosphate a regulator of sugar metabolism in plants? Journal of Experimental Botany 54:533-537.

Elbein AD (1974). The metabolism of, a-trehalose, advance in carbohydrate. Chemistry and Biochemistry 30:227-256.

Folta KM, Maruhnich SA (2007). Green light: a signal to slow down or stop. Journal of Experimental Botany 58(12):3099-3111.

Gibson SI (2000). Plant sugar response pathways. Part of a complex regulatory web. Plant Physiology 124:1532-1539.
Gupta SD, Jatothu B (2013). Fundamentals and applications of lightemitting diodes (LEDs) in vitro plant growth and morphogenesis. Plant Biotechnology Reports 7:211-220.

Hahn EJ, Kozai T, Paek KY (2000). Blue and red light-emitting diodes with or without sucrose and ventilation affect in vitro growth of Rehmannia glutinosa plantlets. Journal of Plant Biology 43:247-250.

Haque SM, Nahar SJ, Shimasaki K (2016). In vitro organogenesis of Anoectochilus formosanaus under different sources of lights. Acta Horticulturae 1134:201-206.

Hesse H, Willmitzer L (1996). Expression analysis of a sucrose synthase gene from sugar beet (Beta vulgaris L.). Plant Molecular Biology 30: 863-872.

Huan LVT, Tanaka M (2004). Effects of red and blue light-emitting diodes on callus induction, callus proliferation, and protocorm-like body formation from callus in Cymbidium orchid. Environmental Control in Biology 42:57-64.

Ichihashi S, Islam MO (1999). Effects of complex organic additives on callus growth in three orchid genera; Phalaenopsis, Doritaenopsis and Neofinetia. Journal of the Japanese Society for Horticultural Science 68(2):269-274.

Kaewjampa N, Shimasaki K (2012). Effects of green LED lighting on organogenesis and superoxide dismutase (SOD) activities in protocorm-like bodies (PLBs) of Cymbidium cultured in vitro. Environmental Control in Biology 50(3):247-254.

Miyashita Y, Kitaya Y, Kubota C, Kozai T, Kimura T (1995). Effects of red and far-red light on the growth and morphology of potato plantlets in vitro: using light emitting diodes as a light source for micropropagation. Acta Horticulturae 393:189-194.

Morrow RC (2008). LED lighting in horticulture. HortScience 43(7):1947-1950.

Nahar SJ, Shimasaki K, Haque SM (2012). Effect of different light and two polysaccharides on the proliferation of protocrom-like bodies of Cymbidium cultured in vitro. Acta Horticulturae 956:307-311.

Nahar SJ, Shimasaki K, Haque SM (2013). Chondroitin sulfate can be a new plant growth regulator for Cymbidium micropropagation. Acta Horticulturae 1014:307-311.

Nahar SJ, Haque SM, Shimasaki K (2014). Rapid PLBs proliferation of Cymbidium spp. in vitro. World Journal of Agricultural Science 10(5):294-299.

Nhut DT, Takamura T, Watanabe H, Okamoto K, Tanaka M (2003). Responses of strawberry plantlets cultured in vitro under superbright red and blue light-emitting diodes (LED). Plant Cell Tissue and Organ Culture 73:43-52.

Schluepmann H, van Dijken A, Aghdasi M, Wobbes B, Paul M, Smeekens S (2004). Trehalose mediated growth inhibition of Arabidopsis seedlings is due to trehalose- 6-phosphate accumulation. Plant Physiology 135(2):879-890.

Seabrook JEA (2005). Light effects on the growth and morphogenesis of potato (Solanum tuberosum) in vitro: a review. American Journal of Potato Research 82(5):353-367.

Shimasaki K, Uemoto S (1990). Micropropagation of a terrestrial Cymbidium species using rhizomes developed from seeds and 
pseudobulbs. Plant Cell Tissue and Organ Culture 22:237-244.

Sultana UH, Shimasaki K, Ahsan MM, Alam MM (2014). Effects of different light quality on growth and development of protocormlike bodies (PLBs) in Dendrobium kingianum cultured in vitro. Bangladesh Research Publications Journal 10(2):223-227.

Sun JD, Nishio JN, Vogelmann TC (1998). Green light drives $\mathrm{CO}_{2}$ fixation deep within leaves. Plant and Cell Physiology 39:10201026.

Tanaka M, Takamura T, Watanabe H, Endo M, Yanagi T, Okamoto $\mathrm{K}$ (1998). In vitro growth of Cymbidium plantlets cultured under superbright red and blue light-emitting diodes (LEDs). Journal of Horticultural Science and Biotechnology 73:39-44.
Tanaka M, Watanabe T, Dam TG, Tanaka M, Takamura T (2001). Morphogenesis in the PLB segments of Phalaenopsis cultured under LED irradiation system (Abstract). Journal of the Japanese Society for Horticultural Science 70:306.

Tang GQ, Luscher M, Sturm A (1999). Antisense repression of vacuolar and cell wall invertase in transgenic carrot alters early plant development and sucrose partitioning. Plant Cell 11:177-189.

Teixeira de Silva JA (2014). Response of hybride Cymbidium (Orchidaceae) protocorm-like bodies to 26 plant growth regulators. Botanica Lithuanica 20(1):3-13.

Wingler A (2002). The function of trehalose biosynthesis in plants. Phytochemistry 60:437-440. 